\title{
Temperature behavior of hot carrier dynamics in InP quantum dots
}

\author{
Artem V. Maleev \\ Institute of Physics, St.-Petersburg State University, St.-Petersburg 198504, Russia \\ Ivan V. Ignatiev* \\ Institute of Physics, St.-Petersburg State University, St.-Petersburg 198504, Russia \\ and Institute of Physics, University of Tsukuba, Tsukuba 305-8571, Japan \\ Il'ya Ya. Gerlovin \\ Vavilov State Optical Institute, St.-Petersburg 190034, Russia \\ Igor E. Kozin ${ }^{\dagger}$ \\ Institute of Physics, St.-Petersburg State University, St.-Petersburg 198904, Russia \\ Yasuaki Masumoto \\ Institute of Physics, University of Tsukuba, Tsukuba 305-8571, Japan \\ (Received 29 August 2004; published 25 May 2005)
}

\begin{abstract}
Photoluminescence (PL) kinetics of the InP self-assembled quantum dots is studied under quasiresonant optical excitation in the temperature range $10-100 \mathrm{~K}$. It is found that the PL rise time abruptly drops with increasing temperature. The model calculations based on the rate equations have shown that this drop cannot result from thermostimulated phonon relaxation of the hot carriers. We developed a model assuming that the main reason for variations in the PL kinetics is related to thermal ejection ("evaporation") of holes from the quantum dots, with a fraction of the dots acquiring negative charge. The model allowed us to describe quantitatively the PL rise as well as the PL decay over the whole temperature range under study. We identified also a number of effects caused by electron evaporation from the quantum dots at temperatures above $60 \mathrm{~K}$. Comparison of the results of the model calculations with the experimental data has allowed us to determine rate parameters of the ejection processes.
\end{abstract}

DOI: 10.1103/PhysRevB.71.195323

PACS number(s): 73.21.La, 78.47.+p, 78.55.Cr

\section{INTRODUCTION}

Semiconductor quantum dots (QDs) have been studied intensely during the recent two decades in connection with their fairly unusual physical properties and their potential use in various optoelectronics devices. ${ }^{1-3}$ A point of great current interest is the possibility of QD application in the so-called spintronics devices making use of the spin orientation effect (spin memory, spin transistors, etc.). ${ }^{4,5}$ The extent to which the QD-based devices come up to expectations depends strongly on the cooling rate of the "hot" carriers created by electric current or by optical excitation, i.e., on the electronphonon relaxation rate.

The hot carrier dynamics in QD structures is made fairly complex by the large scatter of QD parameters in the same sample. The discrete energy spectrum of carriers in a QD imposes stringent constraints upon the possible channels of their relaxation. In QDs in which the energy gap between levels coincides with the energy of one or several longitudinal optical (LO) phonons, carriers relax in times on the order of a few picoseconds. ${ }^{6,7}$ In other QDs carriers have to relax with emission of acoustic phonons. Experimental investigations $^{7}$ and theoretical estimates ${ }^{8-10}$ show this process to be at least two orders of magnitude slower.

The relaxation times of hot carriers are customarily determined by measuring the rise time of the photoluminescence (PL) of electron-hole pairs under pulsed nonresonant optical excitation. At low temperatures (a few Kelvin), where phonon-mediated relaxation is the only possible process, this approach was found to be justified and yielded good results. ${ }^{11-21}$ This stimulated, in its turn, attempts at applying the measured PL rise times to a study of the temperature behavior of the relaxation rate. ${ }^{11,14,19-21}$ High-temperature data are potentially of most interest from the standpoint of applications, because the majority of devices are intended for operation at about room temperature. At the same time, the rich variety of processes is activated in QDs with increasing temperature and interpretation of high-temperature experiments turn out sometimes to be an ambiguous problem. For instance, the shortening of the PL rise time observed experimentally to occur in InAs QDs with increasing temperature was interpreted in Ref. 11 as due to hot carrier relaxation by stimulated phonon emission. In order to reach agreement with experiment, however, the authors had to assume the phonons involved in the relaxation to have energies of about $3 \mathrm{meV}$. At the same time, the energy difference between the absorbed and emitted quanta in their experiment was in excess of $70 \mathrm{meV}$. This discrepancy places the validity of this assumption in some doubt.

We are reporting here on a comprehensive investigation into the temperature-induced variations in the PL kinetics of InP QDs. An analysis of experimental data suggests a conclusion that an increase in temperature activates several dynamic processes, which complicates greatly the general pat- 
tern of the phenomena involved. It was established that the decrease of the PL pulse rise time with increasing temperature originates not from an increase in the relaxation rate but rather from formation of charged QDs, whose PL evolves with substantially different kinetics. We are proposing a model offering a noncontradictory interpretation of the temperature-induced shortening of the PL rise time. The crucial point of the model is the assumption that a photogenerated hole is thermally ejected (evaporated) out of the QD into the barrier. This process accounts for the appearance of charged QDs whose PL has an abrupt pulse leading edge. A model calculation of this process permitted a quantitative description of all experimentally observed temperatureinduced variations in the PL kinetics, or more specifically, the shortening of the PL rise, the decrease of the PL decay time, and the appearance of a long-lived PL component.

\section{EXPERIMENT}

The sample under study is a heterostructure grown on a (100) GaAs substrate by the molecular beam epitaxy (MBE). An InP layer of the nominal thickness of 4 monolayers was grown between 100 -nm-thick $\operatorname{In}_{0.5} \mathrm{Ga}_{0.5} \mathrm{P}$ barrier layers. The average diameter of the QDs, as measured with an atomic force microscope on a reference sample grown in identical conditions but without the top barrier layer, ${ }^{22}$ was $40 \mathrm{~nm}$, and their height was $5 \mathrm{~nm}$. Previous measurements performed on this sample ${ }^{23,24}$ showed the QDs to contain excess charges (electrons). To remove this charge, a small negative bias was applied to the sample surface. For this purpose, a transparent indium-tin oxide electrode was deposited on the sample surface, and an Ohmic contact was attached to the substrate. It was established that at a voltage $U_{\text {bias }}<-0.8 \mathrm{~V}$, InP QDs become neutral. All our PL kinetics measurements were carried out at this voltage.

The PL kinetics was studied with a setup including a Ti:sapphire laser producing 2-ps long pulses at a repetition frequency of $82 \mathrm{MHz}$. The average pump power density on the sample did not exceed $50 \mathrm{~W} / \mathrm{cm}^{2}$. This precluded excitation of more than one electron-hole pair in a QD during one laser pulse and, thus, excluded from consideration the processes not related to phonon-mediated relaxation of hot carriers (the so-called Auger processes ${ }^{20}$ ). PL kinetics measurements at preset spectral points were done by means of a double monochromator with dispersion subtraction (focal length $0.25 \mathrm{~m}$ ) and a Hamamatsu synchroscan streak camera.

The time resolution of the setup was $6 \mathrm{ps}$ when using the fastest streak camera sweep (0-160 ps) and about 15 ps for the slow sweep rate $(0-2000 \mathrm{ps})$.

\section{PL KINETICS}

The QD PL band obtained under nonresonant excitation involving carrier generation in the InGaP barrier layers has a maximum in the 1.7-eV region and a halfwidth of about 50 $\mathrm{meV}$. This halfwidth is primarily caused by statistical scatter of the QD size and shape. The carrier relaxation rate in an InP QD exceeds the recombination rate,$^{20}$ and, therefore, the

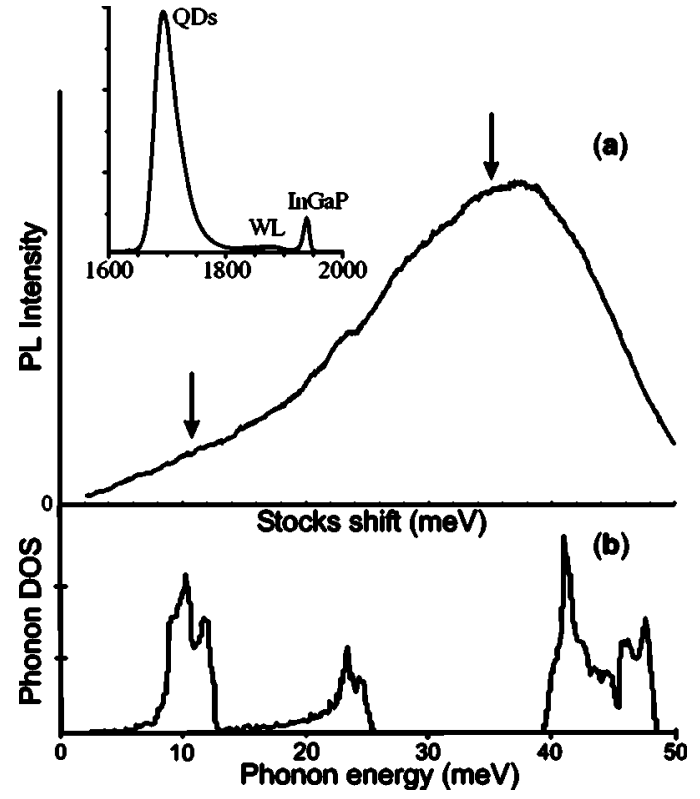

FIG. 1. (a) PL spectrum of InP QDs plotted vs Stokes shift. $E_{\text {exc }}=1765 \mathrm{meV}$. Arrows show the spectral points at which the PL kinetics was studied. (b) Phonon density of states spectrum of InP crystal (Ref. 25). Inset shows the PL spectrum measured at high photon energy of excitation.

PL derives primarily from the lowest state of the electronhole pair.

Nonresonant excitation brings about statistical filling of the QDs by carriers via a complex sequence of carrier capture by QDs and cascade carrier relaxation through intermediate levels, processes which make the pattern of the phenomenon difficult to unravel. Therefore, we chose quasiresonant excitation by radiation with a wavelength slightly above the PL band maximum. This excitation generates electron-hole pairs directly in the quantum dots. As a result, the PL kinetics is determined only by relaxation of the hot carriers to the radiative level, followed by their recombination. Note that the relaxation occurs in one or several steps, depending on the actual energy separation between the excited and radiative states of the electron-hole pair.

Figure 1(a) plots the dependence of PL intensity of the InP QDs on the Stokes shift, $\Delta E_{\text {Stokes }}$, relative to the pump wavelength. The arrows identify the spectral points at which the PL kinetics was measured. The choice of these spectral points was motivated by the specific features of the phonon spectrum (DOS) of InP, which is displayed in Fig. 1(b).

The Stokes shift $\Delta E_{\text {Stokes }}=11 \mathrm{meV}$ (TA spectral point) corresponds to emission in the course of relaxation of one transverse acoustic (TA) phonon. It was shown ${ }^{20}$ that such high-frequency phonons interact efficiently enough with electron-hole pairs. When an electron-hole pair is created 35 $\mathrm{meV}$ above the radiative state (spectral point $2 A C$ ), it can relax with emission of not less than two acoustic phonons, because this energy corresponds to the energy gap between acoustic and optical phonons in the InP crystal. It appears only natural that in this case the relaxation should be slower. As shown below, a comparison of kinetics measurements made at these two spectral points yields valuable information 


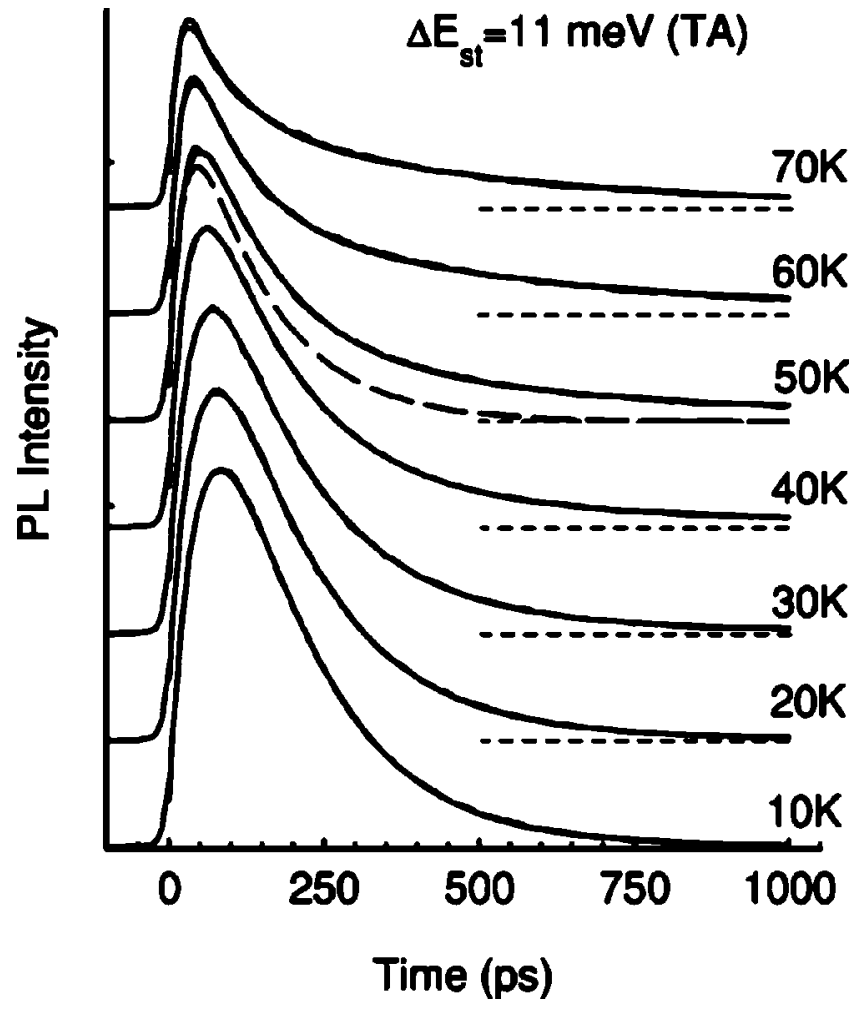

FIG. 2. PL kinetics measured at spectral point TA at different temperatures identified at each curve (noisy curves). For convenience, the curves are translated along the vertical axis. Smooth curves are fits of Eq. (2) to PL kinetics. Note the practically perfect coincidence between the fitting and experimental curves. Dashed curve shows the contribution of the short-lived PL component at $T=50 \mathrm{~K}$.

on the nature of the temperature-induced variations in the PL kinetics of InP QDs.

Figure 2 illustrates characteristic trends in the variation of PL kinetics with increasing temperature. The PL pulse is seen to have a comparatively short leading edge and a longer trailing edge. As the temperature increases, the leading edge shortens, and a slow component appears in the PL decay. In addition, at temperatures above $60 \mathrm{~K}$ a decrease in integrated PL intensity becomes noticeable.

The variation in the shape of the pulse leading edge with increasing temperature is shown in Fig. 3 in expanded scale. It is this variation that was studied by us in the first place. For a preliminary analysis of the temperature-induced kinetics variation we used a simple three-level model. A conventional diagram of the corresponding levels and relevant transitions is displayed in the inset to Fig. 3. We considered the electron-hole pair as a common quantum-mechanical system, whose relaxation rate can be characterized by one parameter. $^{20,21,26}$ By solving standard balance equations for the population of the ground, " 0 ," and excited, "1," states of the electron-hole pair after its excitation by a short pulse, one obtains the evolution of PL intensity with time,

$$
I_{P L}(t)=I_{0}\left(e^{-\gamma_{P L} t}-e^{-\gamma_{r} t}\right),
$$

where $\gamma_{r}=\gamma_{P L}+\gamma_{r e l}$. The quantity $\gamma_{P L}$ is a characteristic of the radiative transition rate from levels 0 and 1 . (The indirect

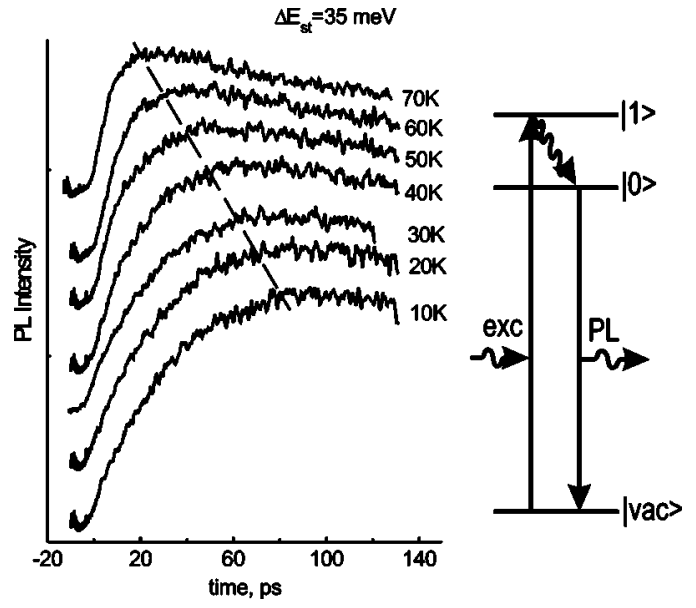

FIG. 3. Initial part of PL kinetics measured at spectral point $2 A C$ at different temperatures identified at each curve. For convenience, the curves are translated along the vertical axis. Dashed line shows the shift of PL pulse maximum with increasing temperature. Inset presents the three-level diagram used in a phenomenological description of PL kinetics.

data quoted in Refs. 19 and 27 show that in a QD these rates are practically equal.) The quantity $\gamma_{r e l}$ defines the relaxation rate from level 1 to level 0 .

At low temperatures, the experimental data could be well fitted by curves drawn using Eq. (1). The fitting parameters were $\gamma_{r e l}, \gamma_{P L}$, and the amplitude factor $I_{0}$. This approach yielded the PL rise and decay times with a good accuracy. Above $40 \mathrm{~K}$, the PL decay becomes nonexponential, with a slow component appearing. To find the duration of this component, we modified Eq. (1) by introducing an additional exponential,

$$
I_{P L}(t)=I_{0}\left[(1-p) e^{-\gamma_{P L} t}+p e^{-\gamma_{s} t}-e^{-\gamma_{r} t}\right],
$$

where factor $p$ characterizes the contribution of the slow component to the PL kinetics, and $\gamma_{s}$ describes its decay rate. As seen from Fig. 2, Eq. (2) approximates quite well the PL kinetics throughout the temperature range covered.

It should be pointed out that, in order to improve the reliability of separation of the fast and slow components, we performed long term accumulation of the PL signal, which permitted us to follow its evolution with time over a large dynamic range. Besides, we made a comprehensive analysis of the totality of experimental data under the assumption of the decay rate of the slow PL component being independent of excitation conditions. (This assumption is validated in Sec. VI.) The separation of the components was favored also by the fact that the rates of their decay differ by a few times throughout the temperature range studied.

Because our main goal consisted in studying the relaxation of electron-hole pairs from the upper excited states, we were interested primarily in the PL rise kinetics. The temperature behavior of the PL rise time, $\tau_{r}=1 / \gamma_{r}$, is displayed for the spectral points TA and $2 A C$ in Figs. 4(a) and 4(b). An increase of the temperature to $100 \mathrm{~K}$ is seen to be accompanied by a considerable decrease in the PL rise time at both spectral points. We are going to analyze in the next section 


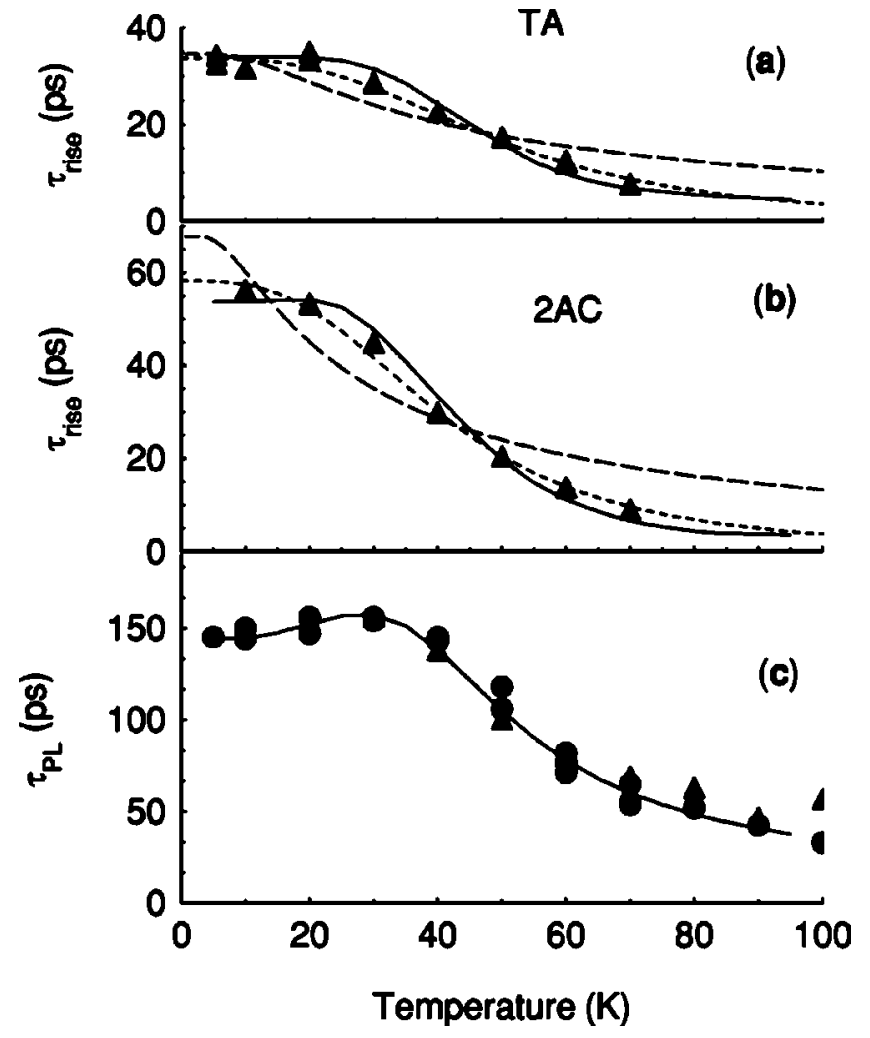

FIG. 4. (a) Temperature behavior of the PL pulse rise time measured at spectral point TA (symbols). The dashed line shows a fit with Eq. (3) made with the model of Ref. 11 (see Sec. IV). Fitting parameters: $E_{p h}=3.2 \mathrm{meV}, \tau_{r e l}(0)=35 \mathrm{ps}$. The dotted line is a fit with Eq. (5) based on the phenomenological model (see Sec. IV). Fitting parameters: $A=(50 \mathrm{~K})^{-3}, \tau_{r e l}(0)=34 \mathrm{ps}$. The solid line is a theoretical curve based on the hole evaporation model (see Sec. V). The model parameters are given in Table I. (b) Same for spectral point 2AC. Fitting parameters for model of Ref. 11, $E_{p h}$ $=1.9 \mathrm{mev}, \tau_{\text {rel }}(0)=68 \mathrm{ps}$; for the phenomenological model, $A$ $=(41 \mathrm{~K})^{-3}, \tau_{r e l}(0)=58 \mathrm{ps}$. (c) Temperature dependence of the fast PL component decay time measured at spectral points TA (circles) and $2 A C$ (triangles). Solid line plots theoretical dependence constructed for the hole evaporation model. The initial part of the graph is approximated by Eq. (15) with parameters $E_{h 01}=4 \mathrm{meV}$ and $\gamma_{P L 1}=(400 \mathrm{ps})^{-1}$.

this decrease quantitatively in terms of phenomenological models which assume the shape of the leading edge of the PL pulse to be governed by relaxation from excited states of the electron-hole pair.

\section{PHENOMENOLOGICAL MODELS OF THERMALLY STIMULATED RELAXATION}

An increase in temperature, i.e., an increase in the energy of thermal vibrations, should, in principle, be accompanied by accelerated relaxation of excited states of the electronhole pair through stimulated phonon emission. Such a process was invoked in Ref. 11 to account for the approximately threefold decrease of the PL rise time in InGaAs QDs with the temperature increasing from 5 to $60 \mathrm{~K}$. In the case of stimulated emission, the temperature dependence of the relaxation time can be written as

$$
\tau_{\text {rel }}(T)=\tau_{\text {rel }}(0) /\left(n_{b}+1\right),
$$

where

$$
n_{b}=\left(e^{E_{p h} / k T}-1\right)^{-1}
$$

is the temperature-dependent population of phonon states with an energy equal to separation between the excited and the lowest sublevels, i.e., with an energy $E_{p h}=E_{12}$, and $k$ is the Boltzmann constant.

The authors of Ref. 11 established that, in the case of InGaAs QDs, Eqs. (3) and (4) describe well the experimental dependence $\tau_{r e l}(T)$ for $E_{p h} \approx 3 \mathrm{meV}$; however the value of $E_{p h}$ obtained is less by more than a factor of 20 than the Stokes shift, i.e., the energy difference between the absorbed and emitted optical quanta (70 $\mathrm{meV}$ or more). In order to account for such a large discrepancy, the authors had to assume that relaxation involves emission of several longitudinal optic (LO) phonons and one longitudinal acoustic (LA) phonon with an energy of $3 \mathrm{meV}$. The specific LA phonon energy was derived from theoretical calculations ${ }^{8}$ which showed that it is such phonons that should interact efficiently with carriers in a QD, because their wavelength is approximately equal to the QD base diameter.

The difference between the energies of the absorbed and emitted quanta in our experiments was always smaller than the LO phonon energy; in other words, LO phonons could not be involved in carrier relaxation. Note that fitting Eqs. (3) and (4) to the experimental temperature dependence of the rise time, with the phonon energy $E_{p h}$ used as a fitting parameter, did not yield positive results. First, as evident from Figs. 4(a) and 4(b), calculated curves disagree with the experiment. Second, the values of the fitting parameter $E_{p h}$ turn out to be substantially smaller than the Stokes shift, which leaves the question of the process accounting for such a fast loss of the remaining electron-hole pair energy unanswered.

In an attempt to modify Eqs. (3) and (4) in such a way as to reach agreement with the experiment we found that the best result is obtained by fitting the experimental data with a function of the kind

$$
\tau_{r e l}(T)=\frac{\tau_{r e l}(0)}{A T^{3}+1}
$$

As seen from Figs. 4(a) and 4(b), the experimental curves are described well by this relation.

A relation of the type of Eq. (5) could ensue if the carrier relaxation were accelerated not by phonons of a specific energy but rather in equal measure by all thermal phonons. Recalling that the major contribution to thermal energy in the temperature interval under study is due to low-frequency acoustic phonons, the phonon spectral density $g(\omega)$ can be approximated by the simple Debye model, ${ }^{28}$ by which

$$
g(\omega)=\frac{3 V \omega^{2}}{2 \pi^{2} v_{0}^{3}},
$$

where $V$ is the cell volume, $\omega$ is the cyclic frequency of acoustic vibrations, and $v_{0}$ is the sound velocity in the material. 
The number of thermal phonons at temperature $T$ is given by the expression

$$
N=\frac{3 V}{2 \pi^{2} v_{0}^{3}}\left(\frac{k T}{\hbar}\right)^{3} \int_{0}^{T_{D} / T} \frac{x^{2} d x}{e^{x}-1} .
$$

At temperatures low compared to the Debye temperature $T_{D}$, the upper limit in Eq. (7) can be replaced by infinity. In this case, Eq. (7) yields

$$
N=A T^{3},
$$

where coefficient $A$ is independent of time and temperature. Substituting Eq. (8) into Eq. (3), we come to the above Eq. (5). Despite a good agreement with the experiment, the mathematical model used does not offer insight into the process of interest, because the assumption of the possibility for a transition at a specific frequency to be stimulated by phonons of the whole thermal spectrum is physically unsubstantiated.

The model of Ref. 11 was used also in Ref. 19 for interpretation of experiments. To reach agreement, the authors had to modify Eq. (3) by replacing the unity in the denominator by a small term $B=0.0039$. No physical validation of this replacement is proposed. ${ }^{19}$ Interestingly, if one eliminates the unity in Eq. (3) altogether, it transfers to the standard expression (the Arrhenius relation) describing the probability for a particle to thermally overcome the barrier.

\section{HOLE "EVAPORATION" MODEL}

The good fit of our experiments with a model taking account of all thermal phonons, as well as the reasonable approximation of the data in Ref. 19 with a relation close to the Arrhenius law, suggest that the shortening of the QD PL leading edge is due not to stimulated emission but rather to absorption of phonons. In other words, an increase in temperature activates the process mediated by a certain potential barrier. This activated process is most probably ejection of a photogenerated hole from a QD. It is known ${ }^{20}$ that the potential well for holes in InP QDs is fairly shallow, $E_{h}$ $\approx 15 \mathrm{meV}$, so that as the temperature becomes high enough thermal phonons will be able to eject the photogenerated hole out of the QD into the barrier. At not too high temperatures $\left(T<E_{h} / k\right)$, the ejection rate should be described by a simple phenomenological relation customarily used to describe a thermally activated process,

$$
\gamma_{h}(T)=\gamma_{h}^{0} e^{-E_{h} / k T},
$$

where $E_{h}$ is the barrier height, and $\gamma_{h}^{0}$ is the rate of the process at a high temperature. ${ }^{29}$

Evaporation of the hole from the QD leaves there an electron, which makes the QD charged. During the interval between laser pulses (about $12 \mathrm{~ns}$ ), this electron drops to the lowest electron level to become "cold". Estimates using the data of Ref. 20 show that the probability for an electron to leave the QD by evaporation or tunneling is much smaller than that for a hole. Therefore, if the hole does not return to the QD, the electron may reside in it until an electron-hole pair is generated there by one of the subsequent laser pulses.
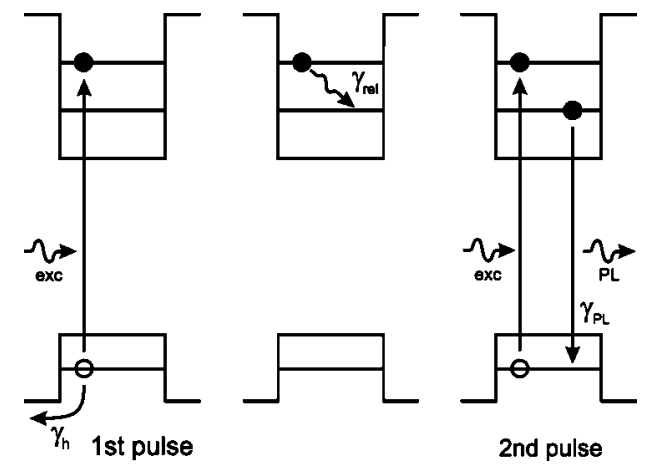

FIG. 5. The scheme of the processes resulting in QD charging. (See details in Sec. V.)

Creation of an electron-hole pair in a charged QD gives rise to the formation of a trion, a triply charged particle. The photogenerated hole in the trion can recombine with the cold electron to produce long-wavelength PL immediately after the laser pulse. This will become manifest in an experiment as a steplike build-up (spike) of the PL in the Stokes region of the spectrum. Such PL spikes are well known for charged QDs. ${ }^{20,21,30}$ Figure 5 displays schematically the sequence of processes bringing about QD charging and excitation of the trion PL.

In a general case, PL of both the neutral and charged QDs are detected. The leading edge of such a pulse has a complex shape, with the PL rise time determined by the relative magnitude of the contributions due to the exciton and trion PL. An increase in temperature will intensify the formation of charged QDs and, hence, reduce the PL rise time.

For pump densities reached usually in experiments, the number of charged QDs produced by one laser pulse is small. Nevertheless, if the electron lifetime in a QD exceeds substantially the laser pulse repetition period, the number of charged QDs will build up to become eventually comparable to that of neutrals. The fractional numbers of charged and neutral QDs, $n_{e}$ and $\left(1-n_{e}\right)$, respectively, are determined by competition between several processes, such as evaporation of the hole and its recombination with the electron, excitation of electron-hole pairs, and escape of the electron from the QD, etc. A comprehensive analysis of these processes given in the Appendix yields the following expression:

$$
\frac{n_{e}}{1-n_{e}}=a \frac{\gamma_{h}(T)}{\gamma_{P L}}
$$

where coefficient $a$ describes the buildup of charged QDs. The buildup is determined by the repetition frequency of laser pulses, their power, and the electron lifetime in a QD.

To describe the PL kinetics, consider the balance equations for the excitons and the trions. In the case of the excitons, the balance equations for the populations of the excited and ground states, $n_{1}^{e x}$ and $n_{0}^{e x}$, contain standard terms describing the photon absorption, relaxation between excited states, and radiative recombination, and also terms responsible for the hole evaporation, 


$$
\begin{aligned}
& \frac{d n_{1}^{e x}}{d t}=\left(1-n_{e}\right) P_{0} \delta(t)-\left[\gamma_{h 1}(T)+\gamma_{r e l}+\gamma_{P L}\right] n_{1}^{e x}, \\
& \frac{d n_{0}^{e x}}{d t}=\gamma_{r e l} n_{1}^{e x}-\left[\gamma_{h 0}(T)+\gamma_{P L}\right] n_{0}^{e x} .
\end{aligned}
$$

Here $P_{0}$ is the probability of photon absorption by a QD. The function $\delta(t)$ takes into account the short duration of the pump pulse compared to the characteristic relaxation times of the processes occurring in a QD. The first line of Eq. (11) also takes account of the fact that excitons can be created only in neutral dots, which make up a $\left(1-n_{e}\right)$ fraction of all dots. The quantities $\gamma_{h 0}(T)$ and $\gamma_{h 1}(T)$ refer to the hole evaporation rates from the excited and ground states of the exciton. As will be shown by an analysis below (Sec. VIII), these rates differ noticeably in the case of the generation of a hot-enough electron-hole pair.

The balance equation for the trions can be written in a similar way,

$$
\begin{aligned}
& \frac{d n_{1}^{t r}}{d t}=n_{e} P_{0} \delta(t)-\left[\gamma_{h t}(T)+\gamma_{r e l}+2 \gamma_{P L}\right] n_{1}^{t r}, \\
& \frac{d n_{0}^{t r}}{d t}=\gamma_{r e l} n_{1}^{t r}-\left[\gamma_{h t}(T)+2 \gamma_{P L}\right] n_{0}^{t r} .
\end{aligned}
$$

Here $n_{1}^{t r}$ is the population of the trion excited state representing a "cold" electron and a photogenerated electron-hole pair in an excited state, and $n_{0}^{t r}$ is the population of the trion ground state, in which both electrons and the hole occupy the lowest levels. In contrast to Eq. (11), in Eq. (12) we neglected the difference between the hole evaporation rates from the ground and excited states. The point is that Coulomb interaction with the second electron increases substantially [by 10-20 meV (Ref. 31)] the well depth for the holes, which makes the energy difference between the hole ground and excited states not very essential. Factor 2 of $\gamma_{P L}$ in Eq. (12) takes into account the possibility of hole recombination with either of the electrons in the trion. ${ }^{32}$

The coupled Equations (11) and (12) yield time dependencies of the exciton and trion state populations, which are needed for calculation of the PL kinetics. The total PL intensity of a sample originating from electron-hole pair recombination both in excitons and in trions is given in a general case by the relation,

$$
I_{P L}(t)=\gamma_{P L} n_{0}^{e x}+2 \gamma_{P L}\left(n_{0}^{t r}+n_{1}^{t r}\right) .
$$

On solving Eqs. (11) and (12), the PL intensity can be recast in the form

$$
\begin{aligned}
I_{P L}(t)= & \gamma_{P L} P_{0}\left[\left(1-n_{e}\right)\left(e^{-\left(\gamma_{h 0}+\gamma_{P L}\right) t}-e^{-\left(\gamma_{h 1}+\gamma_{P L}+\gamma_{r e l}\right) t}\right)\right. \\
& \left.+n_{e}\left(2 e^{-\left(\gamma_{h t}+2 \gamma_{P L}\right) t}-e^{-\left(\gamma_{h t}+2 \gamma_{P L}+\gamma_{r e l}\right) t}\right)\right] .
\end{aligned}
$$

It is Eq. (14) that was used in our simulation of the experimentally measured PL kinetics. The charged QD fraction $n_{e}$ was derived from Eq. (10), and $\gamma_{h i}(T)(i=0,1, t)$ from Eq. (9).
The first term in each of the brackets describes the decay, and the second, the rise of the PL pulse. The temperature dependence of the PL rise rate is determined by the growth in the hole ejection rate $\gamma_{h i}(T)$. This quantity enters the exponent of each of the rising exponentials, as well as determines the temperature behavior of the $n_{e}$ and $\left(1-n_{e}\right)$ population ratio. [See Eq. (10)]. As evident from Eq. (14), the PL kinetics should exhibit a spike at the initial instant of time, $I_{P L}(0)=\gamma_{P L} P_{0} n_{e}$, in which the amplitude is proportional to $n_{e}$ and, thus, depends on $\gamma_{h i}(T)$. Both these factors give rise to the increase of the PL rise rate with temperature.

The PL decay rate should likewise grow with increasing temperature. The increase in the decay rate is due primarily to the evaporation of holes from neutral dots, which is described by the $\gamma_{h 0}(T)$ function in the first exponential in the first bracket. At high temperatures, holes start to evaporate from charged dots, too, thus increasing still more the decay rate.

The temperature behavior of the fast PL component decay time, $\tau_{P L}=\gamma_{P L}^{-1}$, is shown graphically in Fig. 4(c). We readily see that the increase in the rate and, accordingly, the shortening of the PL decay time with increasing temperature are indeed observed experimentally. Note that the decay times observed under excitation at different spectral points coincide practically completely throughout the temperature range covered. This phenomenon allows a simple physical explanation if we take into account that the experimentally measured PL derives from recombination of cold electrons and holes which "forgot" the history of their creation.

Equations (9), (10), and (14) contain several unknown parameters characterizing the rates of carrier relaxation $\left(\gamma_{\text {rel }}\right)$ and recombination $\left(\gamma_{P L}\right)$, the hole evaporation rate from various states $\left(E_{h i}\right.$ and $\left.\gamma_{h i}^{0}\right)$, and the charged QD buildup coefficient $a$. An analysis of two different temperature-dependent effects (PL rise and decay), as well as of the behavior of the long-lived PL component (see the next section), permits one, however, to determine all of them by invoking some additional assumptions.

One can make a priori estimates of the magnitude of some of the parameters. First of all, we assume that carrier relaxation rates do not depend on temperature within the temperature range studied, and therefore, the values of $\gamma_{\text {rel }}$ can be extracted from low temperature measurements. In this way we came to $\gamma_{r e l}=(38 \mathrm{ps})^{-1}$ and $\gamma_{\text {rel }}=(82 \mathrm{ps})^{-1}$ for the spectral points TA and $2 A C$, respectively.

As shown by experiments [see Fig. 4(c)], the PL decay rate grows somewhat (from 145 to $180 \mathrm{ps)} \mathrm{with} \mathrm{temperature}$ in the interval $T=5-30 \mathrm{~K}$, which can apparently be assigned to the thermal population of the excited hole states, which are characterized by a lower radiative recombination rate. ${ }^{33}$ Because these variations are comparatively small, we did not analyze them in any detail and used a simplified relation of the type

$$
\gamma_{P L}(T)=\gamma_{P L 0}\left(1-e^{-E_{h 01} / k T}\right)+\gamma_{P L 1} e^{-E_{h 01} / k T},
$$

which describes the variation of the average probability of recombination for one populated excited state of energy $E_{h 01}$ with respect to the ground state. Here $\gamma_{P L O}$ is the experimen- 
tally determined low-temperature recombination rate, and the energy $E_{h 01}$ and the radiative recombination rate for the excited hole state, $\gamma_{P L 1}$, are fitting parameters.

The hole evaporation process is described by two parameters, more specifically, the prefactor $\gamma_{h}^{0}$ determined by the electron-phonon coupling energy and the potential barrier height $E_{h}$. [See Eq. (9).] Assuming the electron-phonon coupling energy to be approximately the same for the ground and excited states of holes, we used the factor $\gamma_{h}^{0}$ as a common fitting parameter.

While the barrier heights for different hole states are naturally different, they are related fairly rigidly. The barrier height for the hole ground state is equal simply to the depth of the potential well. For the excited state it decreases by the energy of the hot hole. We assume that the excess energy of a hot electron-hole pair, which is determined by the difference between its energies in the excited and ground states and is equal to $\Delta E_{\text {Stokes }}$, divides between the electron and the hole inversely proportionally to their effective masses,

$$
E_{h 1}-E_{h 0}=\Delta E_{\text {Stokes }} m_{e}^{*} /\left(m_{e}^{*}+m_{h}^{*}\right),
$$

where $m_{e}^{*}=0.08$ and $m_{h}^{*}=0.65$ are, accordingly, the effective masses of the electron and the hole. ${ }^{34}$ For charged dots, the barrier height increases by the energy of Coulomb interaction with the second electron and we take the energy of the interaction to be $10 \mathrm{meV}$. Thus, when the potential barriers for different hole states are preset, the only fitting parameter left is the potential well depth, $E_{h 0}$.

One cannot give here an a priori estimate of parameter $a$ describing the buildup of charged QDs, because this effect is governed, as already mentioned, by competition among several processes and, in particular, depends on pump power density. One should therefore expect different measurements to yield different values of $a$, because the pump power density is one of the experimental parameters most difficult to control.

We calculated the temperature-induced variations of the rise and decay times in the initial part of the PL pulse (i.e., disregarding the slow component) in terms of the above model using the same value of $E_{h 0}$ for all experiments. To do this, we used Eq. (14) to calculate the model PL pulse for each spectral point, with the temperatures varied in the interval from 5 to $100 \mathrm{~K}$ in steps of $5 \mathrm{~K}$. The model pulse has a steplike leading edge at elevated temperatures due to buildup of the charged QDs. To determine the rise and decay times for the model PL pulse by the same way as for PL pulse measured experimentally, we (i) convoluted the model PL pulse with the instrument function of the setup, a Gaussian with a halfwidth of $6 \mathrm{ps}$, and (ii) fitted the result using Eq. (1). The parameters $E_{h 0}, \gamma_{h}^{0}$, and $a$ were varied to reach the best agreement with the experiment. It may be added that the parameter $a$ was chosen separately for each spectral point. We should stress that the values of $\tau_{r}$ and $\tau_{P L}$ thus obtained characterize the average rise and decay times of the model PL pulse.

In this way we have succeeded in satisfactorily describing with a single set of parameters the PL pulse shape throughout the temperature range covered. The results of the calculations are plotted by solid lines in Fig. 4. The values of the
TABLE I. Parameters of the main physical processes discussed.

\begin{tabular}{lcl}
\hline \hline Process & Parameter & \multicolumn{1}{c}{ Value } \\
\hline Electron-hole recombination & $\tau_{P L}$ & $145 \mathrm{ps}$ \\
Relaxation of hot carriers & $\tau_{r e l}$ & $38 \mathrm{ps}(\mathrm{TA})$ \\
& & $82 \mathrm{ps}(2 A C)$ \\
Evaporation of holes & $\gamma_{h}^{0}$ & $0.14-0.17 \mathrm{ps}^{-1}$ \\
& $E_{h 0}$ & $15 \mathrm{meV}$ \\
& $E_{h 1}$ & $13.5 \mathrm{meV}(\mathrm{TA})$ \\
& & $11 \mathrm{meV}(2 A C)$ \\
& $E_{h t}$ & $25 \mathrm{meV}$ \\
Evaporation of electrons & $\gamma_{e}^{0}$ & $3-9 \mathrm{ps}^{-1}$ \\
& $E_{e}$ & $55 \mathrm{meV}$ \\
Diffusion of holes in barrier & $\gamma_{s}^{0}$ & $0.002 \mathrm{ps}^{-1}$ \\
Accumulation of charged QDs & $a_{0}$ & $0.4(\mathrm{TA})$ \\
& & $0.35(2 A C)$ \\
\hline \hline
\end{tabular}

model parameters obtained by fitting are listed in Table I. We readily see that the proposed model permits one to quantitatively describe the observed temperature-induced variations in the PL kinetics using physically reasonable assumptions. Note that the value of the parameter $\gamma_{h}^{0}$ characterizing the rate of hole evaporation is approximately higher by a factor of 5 than the relaxation rate of hot carriers $\tau_{\text {rel }}^{-1}$ for the same value of the energy gap. The ratio of the rates thus obtained looks quite reasonable since the hole state density in the barrier substantially exceeds the density of localized states in a QD.

The validity of the proposed model is substantiated by a comprehensive analysis of the shape of the initial part of the PL pulse measured with a high temporal resolution at $T=50 \mathrm{~K}$. (See the inset in Fig. 6.) We readily see that at the initial instant of time one does indeed observe the jump in PL intensity predicted by the theory. To make this jump more revealing, a curve approximating the slow PL rise component is shown (dashed line). Measurements made with a finite time resolution smooth the jump slightly, which is described well by convoluting the theoretical pulse profile with the instrument function.

Figure 6(a) displays also a decomposition of the experimentally measured PL pulse into components deriving from the exciton and trion PL, as well as from the long-lived PL component to be discussed in the next section. One readily sees that all the three components have comparable peak intensities at $T=50 \mathrm{~K}$ but different rise and decay times, which makes the PL pulse shape fairly complex. Figure 6(b) plots calculated temperature dependencies of integrated intensity of the exciton and trion PL. As follows from the graphs, above $60 \mathrm{~K}$ the trion PL becomes stronger than the exciton one.

\section{LONG-LIVED PL COMPONENT}

The hole evaporation process affects the kinetics not only of the PL rise but of the decay as well. A hole ejected into the 


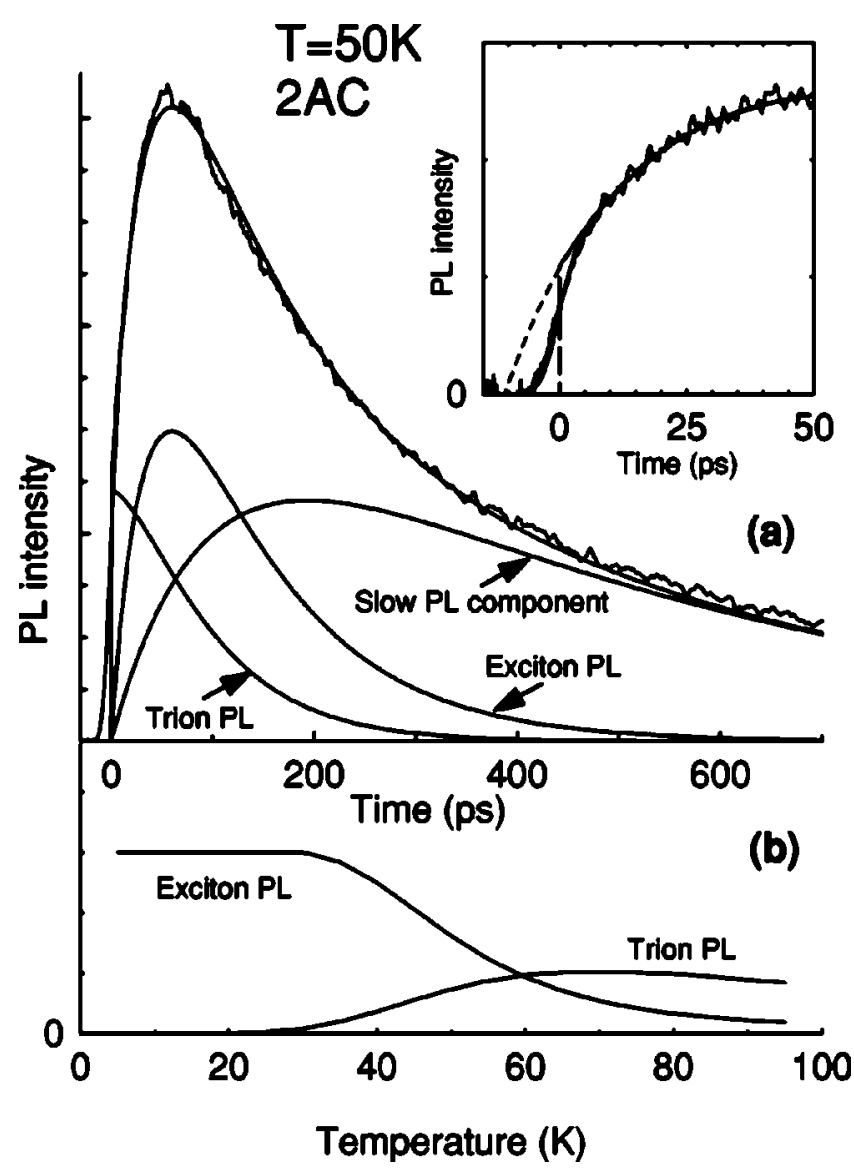

FIG. 6. (a) PL kinetics measured at $T=50 \mathrm{~K}$ at spectral point $2 A C$ (noisy curve). The solid line shows a theoretical curve calculated with the hole evaporation model as a sum of three PL components plotted below. The curve is convoluted with a Gaussian, 6 ps in halfwidth, which simulates the instrument function of the experimental setup. The inset depicts the rising part of the PL pulse (noisy curve). The long-dashed line reproduces instantaneous pulse rise predicted by the model. The short-dashed line depicts pulse evolution with time for the case of the PL being due to exciton luminescence alone. (b) Temperature dependence of integral intensity of the exciton and trion PL.

barrier may after a certain time be retrapped by the QD to recombine with the electron left there. The probability of hole retrapping by a QD is substantially lower than that of its thermal ejection, because the density of hole states in the barrier exceeds by far that in the QD. As a result, the lifetime of a hole in the barrier may turn out to be long enough for its capture by the QD to produce a slow component in the PL kinetics.

To check the validity of this explanation, we analyzed the temperature behavior of integrated intensity of the slow component, $I_{\text {slow }}$, and compared it with model calculations. Because a substantial part of the slow component extended beyond the available detection time interval, we had to extrapolate the PL pulse shape to longer times in order to derive the value of $I_{\text {slow }}$ from experimental data. In view of the fact that the slow component is produced by holes ejected from the QDs, we assumed that the rise time of the slow component is governed by the lifetime of primary holes in a $\mathrm{QD}, \gamma_{P L}^{-1}$, and presented the PL pulse shape in the following form:

$$
\begin{aligned}
I_{P L}(t) & =I_{\text {fast }}(t)+I_{\text {slow }}(t) \\
& =I_{0}\left[\left(e^{-\gamma_{P L} t}-e^{-\gamma_{r} t}\right)+p\left(e^{-\gamma_{s} t}-e^{-\gamma_{P L} t}\right)\right],
\end{aligned}
$$

where the coefficient $p$, which is less than unity, defines the fraction of the slow PL component. Note that Eq. (17) reduces to Eq. (2) by properly rearranging its terms. The quantities $I_{0}, p, \gamma_{P L}, \gamma_{r}$, and $\gamma_{s}$ were used as fitting parameters in a comparison with the experiment. Figure 2 illustrates such a fitting. The fitting parameters determined for each temperature were employed to calculate the ratio of the integrated slow PL component intensity, $I_{\text {slow }}$, to total PL intensity, $I_{\text {total }}$, from the relation,

$$
\frac{I_{\text {slow }}}{I_{\text {total }}}=\frac{p\left(\tau_{s}-\tau_{P L}\right)}{\left(\tau_{P L}-\tau_{r}\right)+p\left(\tau_{s}-\tau_{P L}\right)} .
$$

Equation (18) was obtained by integrating Eq. (17) over time and replacing the rates of the processes involved, $\gamma_{i}$, with their reciprocal quantities, $\tau_{i}=\gamma_{i}^{-1}$.

The results of such treatment of experimental data made for the two spectral points are specified by symbols in Figs. 7 (b) and 7(c). The data are given for $T>20 \mathrm{~K}$ only, because at lower temperatures the intensity of the slow component is too weak to be determined with acceptable accuracy by the above procedure.

We compared these data with model calculations of PL kinetics involving the solution of balance equations for the population of hole states in a QD and the barrier layer. The calculation presented in detail in the Appendix yields the following relation for the ratio of the slow component to total PL intensity:

$$
\frac{I_{\text {slow }}}{I_{\text {total }}}=b \frac{\gamma_{h 1}}{\gamma_{P L}+\gamma_{h 1}} .
$$

Coefficient $b$ in this expression accounts for the hole loss in the barrier which is described in the next section. The temperature dependence of hole evaporation rate from the excited state of a QD, $\gamma_{h 1}$, is described by an expression similar to Eq. (9).

As seen from Figs. 7(b) and 7(c) (dashed curves), Eq. (19) approximates correctly the temperature behavior of the slow PL component up to a temperature of $60 \mathrm{~K}$. Note that the slow component amounts to a considerable fraction of PL at elevated temperatures, namely, up to $80 \%$ at $T=70 \mathrm{~K}$.

Figure 7(d) plots the temperature dependence of integrated PL intensity, $I_{\text {total }}$. We readily see that the total PL intensity practically does not change up to temperatures on the order of $60 \mathrm{~K}$, i.e., all temperature-induced variations occurring in this interval reduce to a redistribution of the fast and slow component intensities. Considered within the model used, this means that all holes injected into the barrier return gradually to the QD to recombine with the electron left there. A further increase of temperature brings about a drop in the total PL intensity. The nature of this effect will be discussed in the next section. 


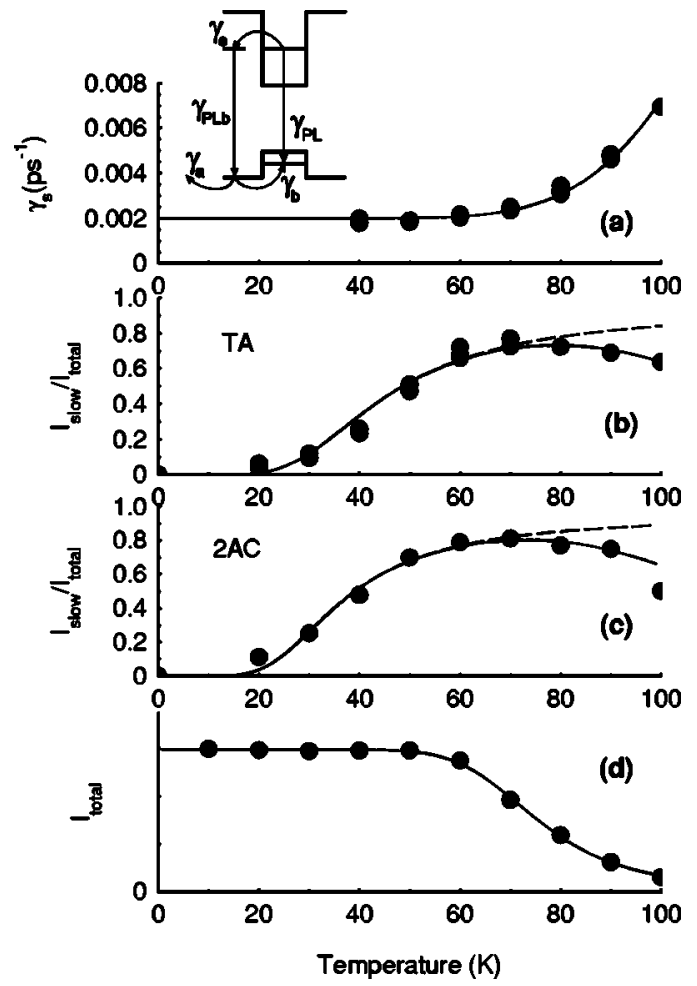

FIG. 7. (a) Decay rate of long-lived PL component (circles). The solid line is a fit with Eq. (20), $\gamma_{e}^{0}=3 \mathrm{ps}^{-1}$. The inset displays schematically the processes involved. (See details in text.) The values of the other parameters used in the calculation are given in Table I. (b) Evolution with temperature of the slow PL component measured at spectral point TA and normalized against total PL intensity (symbols). Dashed line — calculation using Eq. (19) and made neglecting electron evaporation $(b=1)$; solid curve-calculation using Eqs. (19) and (21), $k_{b}=180$. (c) The same for spectral point $2 A C, k_{b}$ $=210$. (d) The temperature dependence of integrated PL intensity measured at spectral point $2 A C$ (circles) and its fitting with Eq. (20) (solid line), $\gamma_{e}^{0}=9 \mathrm{ps}^{-1}$.

\section{THE ROLE OF ELECTRON "EVAPORATION"}

At temperatures $T>60 \mathrm{~K}$, which are comparatively high for the QDs under study, the integrated intensity, $I_{\text {total }}(T)$, falls off noticeably, as seen from Fig. 7(d). The decrease in integrated intensity implies the onset of an additional process leading to nonradiative losses of excitation energy. Because one observes simultaneously a noticeable decrease of the slow component time, which is associated with ejection of holes into the barrier layer [see Fig. 7(a)], it appears only natural to assume that the nonradiative losses occur in the barrier. The most likely loss mechanism involves thermal ejection (evaporation) of a photogenerated electron from the QD with its subsequent recombination with a hole in the barrier. The recombination may either be nonradiative or involve emission of light in a spectral range beyond the spectral range of detection, as this is shown schematically in the inset to Fig. 7(a).

This process can be treated phenomenologically as formation of a channel of electron-hole pair nonradiative relaxation. The relaxation rate in this channel is proportional to the probability of hole recombination with an electron in the barrier. This probability is governed by the electron concentration in the barrier, which grows with temperature as $n_{e}=n_{e}^{0} \exp \left(-E_{e} / k T\right)$. Here $E_{e}$ is the activation energy of electron ejection into the barrier. The appearance of an additional relaxation channel brings about an increase in the decay rate of the slow PL component,

$$
\gamma_{s}(T)=\gamma_{s}^{0}+\gamma_{e}^{0} e^{-E_{e} / k T},
$$

where $\gamma_{s}^{0}$ is the decay rate associated with radiative recombination in the QDs (i.e., the quantity accounting for duration of the slow component at relatively low temperatures), and $\gamma_{e}^{0}$ is a phenomenological constant determined by the density ratio of the electronic states in the barrier and the QD, as well as by the probability of hole recombination with an electron in the barrier.

The solid line in Fig. 7(a) is a plot of Eq. (20) for the parameters $\gamma_{s}^{0}$ and $E_{e}$ listed in Table I and $\gamma_{e}^{0}=3 \mathrm{ps}^{-1}$. This curve is seen to fit well the dependence obtained from the treatment of experimental data. The value of the activation energy derived in this way agrees well with those quoted in Refs. 20 and 35, in which the potential barrier to be surmounted by electrons when ejected from a QD is determined to be about $60 \mathrm{meV}$. This value is substantially less than the potential well depth for electrons in InP QDs, which for the sample under study is $250-300 \mathrm{meV}^{20}$ The relatively low activation energy for the ejection process should be apparently assigned to the electrons being ejected, not into the conduction band of the barrier layer, but onto local defects located in the vicinity of the InP QDs. ${ }^{20,35}$

The existence of a nonradiative relaxation channel brings about a decrease in integrated PL intensity, which is determined by the ratio of the radiative recombination rate of the electron-hole pairs to the total rate of decrease in their population. At temperatures above $60 \mathrm{~K}$, where the slow PL component provides the major contribution to the intensity, the radiative recombination rate is practically equal to $\gamma_{s}$, with the temperature dependence of total intensity taking on the form,

$$
I_{\text {total }}(T)=I_{\text {total }}(0) \frac{\gamma_{s}^{0}}{\left(\gamma_{s}^{0}+\gamma_{e}^{0} e^{-E_{e} / k T}\right)} .
$$

We fitted this equation to experimental data with the values of $\gamma_{s}^{0}$ and $E_{e}$ listed in Table I, with $\gamma_{e}^{0}$ employed as a fitting parameter. As seen from Fig. 7(d), the temperature behavior of $I_{\text {total }}$ is well approximated by Eq. (21).

Hole recombination with electrons in the barrier reduces the intensity of the slow PL component while not affecting in any way that of the fast component, which originates from the recombination of holes before their ejection into the barrier. As a result, at high temperatures the fraction of the slow component should decrease slightly in the high-temperature domain, an effect which is indeed observed in experiment. [See Figs. 7(b) and 7(c).] This effect can be taken into account by including the temperature dependence of coefficient $b$ in Eq. (19), which determines the fraction of the holes retrapped by the QD from the barrier using a phenomenological expression, 


$$
b=\frac{b_{0}}{1+k_{b} e^{-E_{e} / k T}} .
$$

As seen from Figs. 7(b) and 7(c) (solid lines), inclusion of this temperature dependence, with the same activation energy $E_{e}=55 \mathrm{meV}$ as in the preceding approximations, offers a correct description of the behavior of the slow PL component above $60 \mathrm{~K}$ as well.

We had to take into account thermal ejection of electrons into the barrier also when simulating the rise and decay of the fast PL component at high temperatures (see Fig. 6), because it reduces the concentration of charged quantum dots. If electron recombination in the barrier is a fast process, this will increase the total rate of electron escape from the QDs,

$$
\gamma_{e}^{\prime}=\gamma_{e}(0)+\gamma_{e}^{0} e^{-E_{e} / k T},
$$

where the first term describes the rate of electron escape at low temperatures. Equation (22) permits one now to write the relation for the temperature dependence of coefficient $a$ in Eq. (10),

$$
a=\frac{a_{0}}{1+k_{a} e^{-E_{e} / k T}} .
$$

The use of Eq. (23) provides a good fit of the theoretically calculated shape of the fast PL component at various temperatures to experimental results. (See Fig. 6.) The values of parameter $a_{0}$ are listed in Table I for different spectral points, and parameter $k_{a}=300$.

\section{DISCUSSION OF RESULTS}

Our analysis of the PL kinetics revealed that a comparatively small increase in temperature activates in an InP QD structure several processes, which complicate substantially the dynamics of photogenerated carriers. The processes discussed above are depicted schematically in Figs. 5 and 7. The main parameters of the processes are listed in Table I.

The major process which becomes operative already at temperatures $T>30 \mathrm{~K}$ is thermal ejection (evaporation) of a hole from a QD into the barrier layers. This is made possible by the low height of the potential barrier for holes in the structure under study, which is about $15 \mathrm{meV}$. Hole ejection into the barrier gives rise to several effects observed experimentally in the PL kinetics.

If the hole ejected into the barrier does not return to the QD, this QD becomes negatively charged. The kinetics of charged QD PL excited by subsequent laser pulses is characterized by a steep, steplike leading edge resulting from recombination of a photogenerated hole with a cold electron. The model developed here shows that the sharp decrease of the PL rise time observed to occur with increasing temperature can be accounted for primarily by an increase in the contribution to the PL due to luminescence of charged QDs, whose fraction grows with temperature. While competition between various processes of hot carrier relaxation to the radiative level and ejection of the hot hole into the barrier may provide a certain contribution to the shortening of the
PL leading edge, this effect becomes noticeable only at temperatures of the order of $100 \mathrm{~K}$. It should be stressed that the decrease in the PL rise time is in no way related to a decrease in carrier relaxation time, as this is usually assumed. ${ }^{11,19}$ Moreover, we have succeeded in describing quantitatively all the relations observed in this study under the assumption that the hot carrier relaxation rate remains constant within the temperature interval covered.

It should also be emphasized that the decrease of the PL rise time with increasing temperature by the mechanism described above can occur even in the case where $E_{h} \gg k T$, i.e., where each elementary event of hole ejection into the barrier has a low efficiency. For this to be possible, it is sufficient that the lifetime of the electron left in the QD exceeds substantially the pump pulse repetition period. In this case, the fraction of charged QD will grow from one pulse to another, and the buildup parameter $a$ in Eq. (10) may turn out large enough to compensate for the low evaporation rate $\gamma_{h}$.

Another consequence of hole ejection into the barrier is the decrease of the fast PL component decay time, which is also described quantitatively within the model developed here. The third effect is the appearance at elevated temperatures of a long-lived PL component, which originates from the holes being retrapped by the parent QDs. This effect also allows a good description in terms of a common model. Finally, a qualitative argument for the validity of the model comes from the presence at the leading edge of the PL pulse of two distinctly separated regions, namely, a fast (charge QD luminescence) and a relatively slow (neutral QD luminescence) one. We believe that all these effects provide a convincing argument for hole evaporation being a major cause of the strong modification of PL kinetics with increasing temperature.

This analysis revealed also some effects originating from electron evaporation (Sec. VII). In the structures under study, these effects are observed at higher temperatures $(T>60 \mathrm{~K})$ than those associated with the evaporation of holes, which should be assigned to the potential barrier for this process being higher $\left(E_{e}=55 \mathrm{meV}\right)$.

We believe that the processes considered in the present study, rather than being specific of the structure investigated here, are of a fairly general nature. One cannot, in particular, exclude the possibility that the decrease of the PL rise time observed to occur with increasing temperature in Refs. 11,14 and 19 likewise derives from a change in the fractional content of charged and neutral QDs. One should, however, bear in mind that the potential well depths for electrons and holes in InAs QDs investigated by the above authors differ to a lesser extent, and, therefore, identifying the first process to become activated with increasing temperature, more specifically, evaporation of electrons or holes, would require a separate study.

The results obtained in our study suggest that, in order to reliably identify the processes accounting for the PL rise in QDs, one has to carry out a comprehensive investigation of the temperature behavior not only of the leading but of the trailing PL pulse edges as well. Most of the publications dealing with carrier relaxation do not analyze in detail the temperature dependence of the PL kinetics, in particular, they do not separate PL into fast and slow components, although 
some authors (see, e.g., Refs. 13,19 and 36) make a mention of an increase in the PL decay time with increasing temperature, while Marcinkevičius and Leon, ${ }^{14}$ on the contrary, reports a decrease of this time. It is conceivable that one of the reasons for the discrepancies in the final results between these studies (aside from differences between the samples used) consists in an inadequate investigation of the PL decay kinetics.

It should be emphasized that, because of differences in the kinetics of neutral and charged QDs, the PL pulse shape may depend appreciably on the number of charged QDs in the sample. There are a lot of processes capable of changing the QD charge. In particular, when exciting PL with a large Stokes shift, ${ }^{14}$ heating of the sample may change the mobility of photogenerated carriers in the barrier layer or the built-in electric field. Residual background doping of the barrier layers may bring about partial charging of QDs even at a low temperature. ${ }^{37}$ As a result, the number of charged QDs may become dependent both on the doping level and on the pump conditions and sample temperature. It is probably the diversity of the above effects that accounts for variation of the available literature data on characteristic PL rise times in structures of essentially the same type from a few tens of picoseconds ${ }^{14,15,17,18}$ to a few nanoseconds. ${ }^{19}$

\section{CONCLUSION}

Our study of the temperature behavior of PL kinetics in InP QDs has permitted identification of several hot-carrier dynamic processes which result in a strong change of the PL pulse shape. It was established that the decrease of the PL pulse rise time observed to result from a comparatively small increase of temperature from 10 to $100 \mathrm{~K}$ is caused by thermally stimulated ejection ("evaporation") of holes out of QDs, which makes part of the dots negatively charged. Recombination of a hole generated by a subsequent laser pulse with an electron present in the QD gives rise to a PL spike at a wavelength shifted relative to that of the laser line. As the temperature increases, the fraction of charged QDs grows, thus reducing the average rise time of the PL pulse. Our studies did not reveal any noticeable acceleration of hot carrier relaxation in the temperature interval covered, although it is this process that is used by many authors to account for the decrease in the QD PL rise time at elevated temperatures. Hole evaporation brings about also a decrease of the PL decay time, and the return of ejected holes back to the QD is responsible for the appearance of a long-lived component in PL kinetics. The fraction of this component builds up to $80 \%$ at a temperature of $70 \mathrm{~K}$. We identified also a number of effects caused by electron evaporation from QDs.

\section{ACKNOWLEDGMENTS}

The authors thank Professor V.F. Agekyan for fruitful discussion. Experimental data throughout this work were taken in the Masumoto Single Quantum Dot Project, ERATO, JST which authors greatly acknowledge. This work is partially supported by the Russian Foundation for Basic Research (project No. 0302-16858), by INTAS (project No. 1B 2167), and by ISTS (project No. 2679).

\section{APPENDIX}

Consider in more detail the evaporation dynamics of photogenerated holes out of neutral QDs. We are going to consider one hole state in the QD, with the population denoted by $n_{x}$, and one hole state in the barrier layer, with the population $n_{b}$. The population dynamics of these states is given by the equations

$$
\begin{aligned}
& \frac{d n_{x}}{d t}=\left(1-n_{e}\right) P_{0} \delta(t)-\left(\gamma_{h}+\gamma_{P L}\right) n_{x}+\gamma_{b} n_{b}, \\
& \frac{d n_{b}}{d t}=\gamma_{h} n_{x}-\left(\gamma_{a}+\gamma_{b}+\gamma_{e}\right) n_{b} .
\end{aligned}
$$

The first of these equations describes a generation of a hole in an uncharged QD (the first term), escape of the hole from the QD through its recombination with an electron or evaporation (the second term), and return of the hole from the barrier back to the QD. The second equation considers migration of the hole into the barrier out of the QD (first term) and its escape out of the barrier (second term) via transfer to another QD at a rate $\gamma_{a}$, return back to the "parent" QD at a rate $\gamma_{b}$, or hole recombination with the electron which had evaporated from the QD at a rate $\gamma_{e}$. We are going to assume in what follows that the probability for a hole to transfer to another QD (a process giving rise to formation of charged QDs) is substantially lower than that to return to the parent dot, i.e., that $\gamma_{a} \ll \gamma_{b}$.

Equation (A1) for the hole state population in a QD can be solved to yield

$n_{x}=\left(1-n_{e}\right) P_{0} e^{-x_{1} t}+\left(1-n_{e}\right) P_{0} \frac{1}{2}\left(1-\frac{\gamma_{1}-\gamma_{s}}{\sqrt{D}}\right)\left(e^{-x_{2} t}-e^{-x_{1} t}\right)$,

where

$$
\begin{aligned}
& x_{1}=\frac{1}{2}\left[\left(\gamma_{1}+\gamma_{s}\right)+\sqrt{D}\right], \\
& x_{2}=\frac{1}{2}\left[\left(\gamma_{1}+\gamma_{s}\right)-\sqrt{D}\right],
\end{aligned}
$$

with the following notation: $D=\left(\gamma_{1}-\gamma_{s}\right)^{2}+4 \gamma_{h} \gamma_{b}, \gamma_{1}=\gamma_{P L}$ $+\gamma_{h}, \gamma_{s}=\gamma_{a}+\gamma_{b}+\gamma_{e}$.

The PL intensity of uncharged QDs is given by the expression $I_{P L}=\gamma_{P L} n_{x}$. The first term in Eq. (A2a) describes decay of the fast PL component, and the second, both the rise and decay of the slow PL component. Note that the decay rates of these components, $x_{1}$ and $x_{2}$, differ from those used in the text of the paper. (See Secs. VI and VII.) This difference is caused by the interrelation of the equations for the populations $n_{x}$ and $n_{b}$ through the rates $\gamma_{h}$ and $\gamma_{b}$. [See Eqs. (A1).] At not too high temperatures, however, at which $\gamma_{b}$ is substantially smaller than $\gamma_{h}$ and $\gamma_{P L}$, this difference is small 
and vanishes in linear-in- $\gamma_{b}$ approximation for $\sqrt{D}$ which we shall use subsequently. This approximation yields the relations employed in the main text,

$$
x_{1} \approx \gamma_{P L}+\gamma_{h}, \quad x_{2} \approx \gamma_{b}^{e f f}+\gamma_{e},
$$

where

$$
\gamma_{b}^{e f f}=\gamma_{b} \frac{\gamma_{P L}}{\gamma_{P L}+\gamma_{h}} .
$$

Note that the quantity $\gamma_{b}^{e f f}$ can depend on temperature in a complex way. In the low-temperature domain it can decrease as a result of hole localization in shallow potential wells in the barrier, which is described by the temperature dependence of parameter $\gamma_{b}$. At elevated temperatures it can fall off through efficient ejection of holes out of QDs back into the barrier, a process specified by the fraction in Eq. (A3b). As mentioned in Sec. VII experimental data are satisfactorily approximated by assuming $\gamma_{b}^{e f f}=\gamma_{s}^{0}=$ const, which is possibly due to the above processes canceling one another in the temperature interval under study.

Integrated intensity of the slow PL component for uncharged QDs can be calculated by integrating the second term in Eqs. (A2a) and (A2b) over time in the linear-in- $\gamma_{b}$ approximation,

$$
I_{\text {slow }}^{x}=\left(1-n_{e}\right) P_{0}\left(\frac{\gamma_{h}}{\gamma_{P L}+\gamma_{h}}\right)\left(\frac{\gamma_{b}^{e f f}}{\gamma_{b}^{e f f}+\gamma_{e}}\right) .
$$

For charged QDs one can write, in principle, dynamic equations similar to Eq. (A1), with replacements $\left(1-n_{e}\right)$ $\rightarrow n_{e}, \gamma_{h} \rightarrow \gamma_{h t}$, and $\gamma_{P L} \rightarrow 2 \gamma_{P L}$, and solve to obtain similar solutions. The evaporation rate of holes from charged QDs at temperatures $T<60 \mathrm{~K}$ is, however, appreciably lower than that from neutral ones $\left(\gamma_{h t} \ll \gamma_{h}\right)$ because of the hole being additionally bound by Coulomb forces to the second electron. Therefore integrated intensity of the slow component for charge dots, $I_{\text {slow }}^{\text {tr }}$, should be, according to an equation similar to (A4), low. At elevated temperatures, evaporation of one of the electrons results in an abrupt decrease of the potential well depth for the holes, thus making their evaporation as efficient as that from neutral QDs. Therefore at these temperatures the slow PL component of charged QDs should be described by a relation similar to Eq. (A4) but with the replacement $\left(1-n_{e}\right) \rightarrow n_{e}$. The expression for total PL intensity, $I_{\text {slow }}=I_{\text {slow }}^{x}+I_{\text {slow }}^{\text {tr }}$, is derived from Eq. (A4) by re- moving the $\left(1-n_{e}\right)$ factor. Note that evaporation of an electron from a neutral dot also entails a decrease of the potential well depth for the hole, thus making hole evaporation from the dot still more efficient. This does not, however, increase the slow component intensity, because the return of the hole to the QD is not accompanied by PL as there is no electron there.

As pointed out in the main text (see Sec. VII), thermal ejection of an electron into the barrier and its recombination with a hole give rise to quenching of the QD PL. Straightforward description of this process is made difficult by the above-mentioned abrupt change in the hole evaporation rate. Therefore, we shall restrict ourselves to a phenomenological description of the PL quenching process presented in the main text.

Solving Eqs. (A1) yields also the dynamics of the hole state population in the barrier,

$$
n_{b}=\left(1-n_{e}\right) P_{0} \frac{\gamma_{h}}{\sqrt{D}}\left(e^{-x_{2} t}-e^{-x_{1} t}\right) .
$$

Escape of a hole from the barrier layer to another QD or to a defect, which is described by a rate $\gamma_{a}$, brings about longlived charging of the QD out of which the hole has been ejected. The increase of the probability for a QD to become charged during the laser pulse period, $\Delta n_{e p}$, can be calculated by integrating Eq. (A5) over time and multiplying the result obtained by $\gamma_{a}$,

$$
\Delta n_{e p}=\gamma_{a} \int n_{b} d t \approx\left(1-n_{e}\right)\left[\left(P_{0} \frac{\gamma_{a}}{\gamma_{b}}\right) \frac{\gamma_{b}^{e f f}}{\gamma_{b}^{e f f}+\gamma_{e}}\right] \frac{\gamma_{h}}{\gamma_{P L}} .
$$

Equation (A6) was derived under the same assumptions as Eqs. (A3a) and (A3b).

During the time between pulses, QDs discharge at a rate $\gamma_{e}$. Assuming the variation in the fraction of charged dots after each pulse to be small compared to the average value, i.e., $\Delta n_{e} \ll n_{e}$, we conclude that QDs discharge exponentially, with $n_{e}$ decreasing during the time between successive pulses by an amount $\Delta n_{e m} \approx n_{e} \gamma_{e} / f$, where $f$ is the laser pulse repetition frequency. When pumped in the quasistationary mode, the fraction of charged dots acquires an equilibrium level found from the condition $\Delta n_{e p}=\Delta n_{e m}$. This condition, together with Eq. (A6), yields Eqs. (10) and (23) in the main text of the paper.

\footnotetext{
*Electronic address: ignatiev@pobox.spbu.ru

†Present address: TurboDisk, Veeco Instruments Inc., 394 Elizabeth Ave., Somerset, NJ 08873.

${ }^{1}$ D. Bimberg, M. Grundmann, and N. N. Ledenstov, Quantum Dot Heterostructures (Wiley, New York, 1999).

${ }^{2}$ M. Sugawara, Self-Assembled InGaAs/GaAs Quantum Dots, Semiconductors and Semimetals Vol. 60 (Academic Press, Orlando, FL, 1999).

${ }^{3}$ Semiconductor Quantum Dots. Physics, Spectroscopy and Appli-
}

cations, edited by Y. Matsumoto and T. Takagahara, Springer Series in NanoScience and Technology (Springer-Verlag, Berlin, 2002).

${ }^{4}$ D. Loss and D. P. DiVincenzo, Phys. Rev. A 57, 120 (1998); D. D. Awschalom and J. M. Kikkawa, Phys. Today 52 (6), 33 (1999).

${ }^{5}$ D. D. Awschalom, D. Loss, and N. Samarth, Semiconductor Spintronics and Quantum Computation (Springer-Verlag, Berlin, 2002). 
${ }^{6}$ S. A. Permogorov, Phys. Status Solidi B 68, 9 (1975).

${ }^{7}$ V. F. Gandmacher and Y. B. Levinson, Carrier Scattering in Metal and Semiconductors (North-Holland, Amsterdam, 1987).

${ }^{8}$ U. Bockelmann and G. Bastard, Phys. Rev. B 42, 8947 (1990).

${ }^{9}$ H. Benisty, C. M. Sotomayor-Torres, and C. Weisbuch, Phys. Rev. B 44, 10945 (1991).

${ }^{10}$ T. Inoshita and H. Sakaki, Phys. Rev. B 46, 7260 (1992).

${ }^{11}$ B. Ohnesorge, M. Albrecht, J. Oshinowo, A. Forchel, and Y. Arakawa, Phys. Rev. B 54, 11532 (1996).

${ }^{12}$ M. Vollmer, E. J. Mayer, W. W. Ruhle, A. Kurtenbach, and K. Eberl, Phys. Rev. B 54, R17292 (1996).

${ }^{13}$ M. Braskén, M. Lindberg, M. Sopanen, H. Lipsanen, and J. Tulkki, Phys. Rev. B 58, R15993 (1998).

${ }^{14}$ S. Marcinkevic ius and R. Leon, Phys. Rev. B 59, 4630 (1999).

${ }^{15}$ D. Morris, N. Perret, and S. Fafard, Appl. Phys. Lett. 75, 3593 (1999)

${ }^{16}$ C. Lobo, N. Perret, D. Morris, J. Zou, D. J. H. Cockayne, M. B. Johnston, M. Gal, and R. Leon, Phys. Rev. B 62, 2737 (2000).

${ }^{17}$ S. Lan, K. Akahane, H. Z. Song, Y. Okada, M. Kawabe, T. Nishimura, and O. Wada, Phys. Rev. B 61, 16847 (2000).

${ }^{18}$ S. Marcinkevičius, A. Gaarder, and R. Leon, Phys. Rev. B 64, 115307 (2001).

${ }^{19}$ R. Heitz, H. Born, F. Guffarth, O. Stier, A. Schliwa, A. Hoffmann, and D. Bimberg, Phys. Rev. B 64, 241305(R) (2001).

${ }^{20}$ I. V. Ignatiev, I. E. Kozin, V. G. Davydov, S. V. Nair, J. S. Lee, H. W. Ren, S. Sugou, and Y. Masumoto, Phys. Rev. B 63, 075316 (2001).

${ }^{21}$ I. V. Ignatiev and I. E. Kozin, in Semiconductor Quantum Dots. Physics, Spectroscopy and Applications (Ref. 3), p. 245.

${ }^{22}$ H. W. Ren, M. Sugisaki, S. Sugou, K. Nishi, A. Gomyo, and Y. Masumoto, Jpn. J. Appl. Phys., Part 1 38, 2438 (1999).

${ }^{23}$ I. E. Kozin, V. G. Davydov, I. V. Ignatiev, A. V. Kavokin, K. V. Kavokin, G. Malpuech, H. W. Ren, M. Sugisaki, S. Sugou, and Y. Masumoto, Phys. Rev. B 65, 241312(R) (2002).

${ }^{24}$ I. A. Yugova, I. Ya. Gerlovin, V. G. Davydov, I. V. Ignatiev, I. E. Kozin, H. W. Ren, M. Sugisaki, S. Sugou, and Y. Masumoto, Phys. Rev. B 66, 235312 (2002).

${ }^{25}$ Phonon density of bulk InP at room temperature is calculated by P. W. Yu, D. N. Talwar, H. Q. Hou, and C. W. Tu, Phys. Rev. B 49, 10735 (1994). In the InP QDs, the phonon spectrum is slightly modified due to the compress strain of the InP crystal lattice in the heterostructure. ${ }^{20}$ To consider this modification and the temperature effect, we expanded the calculated spectrum by multiplying the energy scale with factor 1.07 and shifted the acoustic maxima by $1 \mathrm{meV}$.

${ }^{26}$ O. Verzelen, R. Ferreira, and G. Bastard, Phys. Rev. Lett. 88, 146803 (2002).

${ }^{27}$ S. Raymond, X. Guo, J. L. Merz, and S. Fafard, Phys. Rev. B 59, 7624 (1999).

${ }^{28}$ C. Kittel, Introduction to Solid State Physics (Wiley, New York, 1995).

${ }^{29}$ A more complicated process is possible. As it is discussed in Ref. 21 and also by A. Vasanelli et al., Phys. Rev. Lett. 89, 216804 (2002); R. Oulton et al., Phys. Rev. B 68, 235301 (2003), the energy spectrum of the electron-hole pair in a QD is overlapped with the states when one of the carriers (say electron) is created at the QD ground state and the other one (say hole) is created at a state of continuum of the barrier or wetting layer states. Due to this overlap, the hole can escape from the dot even at a low temperature if the excess energy of the photocreated electronhole pair over its lowest energy exceeds the potential energy of the hole in the QD. This process can be accelerated with temperature; however this is not studied theoretically. Nevertheless, we believe that this process is less efficient than that described in the main text because, according to our experimental results, an increase of the excess energy results in the slowing down rather than acceleration of the hole escape as it is evident from comparison of the data for the TA and $2 A C$ spectral points.

${ }^{30}$ Y. Masumoto, I. V. Ignatiev, I. E. Kozin, V. G. Davydov, S. V. Nair, J. S. Lee, H. W. Ren and S. Sugou, Jpn. J. Appl. Phys., Part 1 40, 1947 (2001).

${ }^{31}$ S. Nair, in Semiconductor Quantum Dots. Physics, Spectroscopy and Applications (Ref. 3), p. 439.

${ }^{32}$ Electron and hole spin degrees of freedom impose certain selection rules on recombination channels for both excitons and trions. In the case of excitons, the electron and the hole recombine if their spins are antiparallel ("bright" states of the exciton) and cannot recombine for parallel spins ("dark" exciton states). In the case of cold trions, with electrons in the ground state and their spins paired, the hole recombines with one of the electrons for any orientation of its spin. The spins of electrons and holes in a low-temperature sample are coupled by exchange interaction [binding energy of about $3 \mathrm{meV}$ (Ref. 20)]. The exciton PL is determined in this case by exciton spin dynamics, which depend on the QD anisotropy and excitation conditions and can be fairly complex. As the temperature is increased, however, the exchange coupling of the electron and hole spins breaks, and the exciton may reside with equal probability in the bright and dark states. Unlike the exciton, the trion is always in the emitting state, because it remains cold at the sample temperatures covered in the present study. This is what accounts for the twofold difference in recombination rates between the excitons and the trions. A more comprehensive discussion of the spin effects would be outside the scope of this communication.

${ }^{33}$ Another possible explanation for the increase in the electron-hole pair recombination time could be thermally activated hole spin flip. When involved in this process, the electron-hole pair resides part of the time in a nonemitting state.

${ }^{34}$ Semiconductors, Intrinsic Properties of Group V Elements and III-V, II-VI, and I-VII Compounds, edited by K.-H. Hellwege and O. Madelung, Landolt-Bornstein, New Series, Group III, Vol. 22, Pt. A (Springer-Verlag, Berlin, 1987).

${ }^{35}$ I. V. Ignatiev, I. E. Kozin, H. W. Ren, S. Sugou, and Y. Masumoto, Phys. Rev. B 60, R14001 (1999).

${ }^{36}$ F. Adler, M. Geiger, A. Bauknecht, D. Haase, P. Ernst, A. Dornen, F. Scholz, and H. Schweizer, J. Appl. Phys. 83, 1631 (1998).

${ }^{37}$ A. Hartmann, Y. Ducommun, E. Kapon, U. Hohenester, and E. Molinari, Phys. Rev. Lett. 84, 5648 (2000). 